\title{
COUGH
}

\section{Antitussive activity of iodo-resiniferatoxin in guinea pigs}

\author{
M Trevisani, A Milan, R Gatti, A Zanasi, S Harrison, G Fontana, A H Morice, P Geppetti
}

See end of article for

Thorax 2004;59:769-772. doi: 10.1136/thx.2003.012930

authors' affiliations

....................

Correspondence to: $M$ Trevisani, PhD,

Center of Excellence for the

Study of Inflammation,

University of Ferrara,

44100 Ferrara, Italy;

tvm@unife.it

Received 17 July 2003

Accepted 10 March 2004
Background: lodo-resiniferatoxin (I-RTX) has recently been described as an ultra potent antagonist of the transient receptor potential vanilloid-1 (TRPV1).

Methods: The ability of I-RTX to inhibit cough induced by inhalation of two putative TRPVI stimulants (capsaicin and citric acid) was tested in non-anaesthetised guinea pigs.

Results: Pretreatment with I-RTX either intraperitoneally $(0.03-0.3 \mu \mathrm{mol} / \mathrm{kg})$ or by aerosol $(0.1-3 \mu \mathrm{M})$ reduced the number of coughs produced by inhalation of citric acid $(0.25 \mathrm{M})$ and capsaicin $(30 \mu \mathrm{M})$ in a dose dependent manner. Capsazepine (CPZ) also reduced citric acid and capsaicin induced cough, but the activity of I-RTX was 10-100 times more potent than CPZ in all the experimental conditions tested. Conclusions: I-RTX is a novel and potent antitussive drug which inhibits cough mediated by agents possibly acting via TRPVI activation.
C ough is one of the most common reasons for medical consultation. In most cases, however, drugs currently available for antitussive treatment are only partially effective. In the search for safe and effective antitussive drugs it has been noted that, in some animal models and in humans, tussigenic agents stimulate a recently cloned ion channel belonging to the transient receptor potential family of channels. ${ }^{1}$ Since this entity is activated by molecules with a vanilloid moiety it has been termed the vanilloid receptor-1 (VRI), and more recently reclassified as the transient receptor potential vanilloid-1 (TRPVI). ${ }^{2}$

TRPVI is stimulated by several different agents including heat $\left(43-52^{\circ} \mathrm{C}\right),{ }^{1}$ protons, ${ }^{3}{ }^{4}$ anandamide, ${ }^{5}$ and other lipid derivatives.$^{67}$ Capsaicin, a well known stimulant of TRPVl, is frequently used in provocation tests to induce cough in experimental animal models and also in man..$^{8-10}$ Citric acid is also widely used for this purpose. The observation that capsazepine (CPZ), a relatively selective but low potency TRPVI antagonist, reduced both capsaicin and citric acid induced cough suggested that TRPVl is involved in the tussive response induced by these two agents. ${ }^{11}$ A lowered threshold to capsaicin induced cough seems to be linked to the sex of the patient ${ }^{12}$ and has been seen in patients with chronic inflammatory airway diseases including asthma ${ }^{13}$ and chronic obstructive pulmonary disease (COPD). ${ }^{14}$ TRPVl therefore seems to have a role in the physiological activation of the cough reflex, as well as in the exaggerated cough response observed during certain pathological states.

On this basis it can be predicted that TRPVI antagonists are of therapeutic value not only in the treatment of cough in patients with asthma and COPD, but also in patients with other inflammatory diseases including post viral cough and cough related to gastro-oesophageal reflux where upregulation of TRPVl sensitivity has been implicated. ${ }^{15}$ However, the TRPVI antagonists currently available suffer from poor specificity or potency. For instance, ruthenium red selectively inhibits TRPVl in a very narrow range of concentrations ${ }^{16}$ whereas, because of its low potency, CPZ effectively blocks it only at high concentrations. ${ }^{17}$ It has recently been reported that iodo-resiniferatoxin (I-RTX), the iodinated form of the ultra potent TRPVl agonist resiniferatoxin, ${ }^{18}$ behaves as a high affinity TRPVl antagonist at the mouse and rat recombinant TRPVI. ${ }^{19}$ We have confirmed this early observation by showing that, in a series of "typical" nociceptive or neurogenic inflammatory responses activated by capsaicin,
I-RTX behaves as an ultra potent antagonist at the native rat and guinea pig TRPVl and at the recombinant human TRPVI. ${ }^{20}$

The aim of the present study was to investigate whether IRTX can reduce capsaicin and citric acid induced cough in guinea pigs and to compare its potency with that of CPZ.

\section{METHODS \\ Animals}

Male Dunkin-Hartley guinea pigs (250-350 g, Pampaloni, Italy) were acclimatised in cages at a mean (SD) temperature of $24(0.5)^{\circ} \mathrm{C}$ ) for 1 week after delivery and were allowed free access to water and standard rodent diet (Morini, Italy). All experiments complied with the national guidelines and were approved by the regional ethics committee.

\section{Experimental set up}

After acclimatisation to laboratory conditions, animals were individually placed in a transparent perspex box $(20 \times 10 \times 10 \mathrm{~cm}$, Vetrotecnica, Italy $)$ ventilated with a constant airflow of $400 \mathrm{ml} / \mathrm{min}$. The tussive agents (citric acid $0.25 \mathrm{M}$ and capsaicin $30 \mu \mathrm{M}$ ) were nebulised via a miniultrasonic nebuliser (Ugo Basile, Italy). The particle size produced had an aerodynamic mass median diameter of $0.9 \mu \mathrm{m}$ and the output of the nebuliser was $0.4 \mathrm{ml} / \mathrm{min}$. The appearance of cough was detected by means of a tie clip microphone (Sony, Japan) and confirmed by the characteristic posture of the animal. The cough sounds were recorded and digitally stored. The number of elicited cough efforts was subsequently counted by a blinded observer.

\section{Study protocols}

All experiments were carried out at the same time of day starting at 09.00 hours. The guinea pigs were exposed to aerosols of either capsaicin or citric acid for 10 minutes to elicit cough. To evaluate the effects of aerosolised I-RTX on experimentally induced cough, guinea pigs inhaled various concentrations of I-RTX corresponding to $0.3,1$, and $3 \mu \mathrm{M}$ in capsaicin trials and $0.1,0.3$, and $1 \mu \mathrm{M}$ in citric acid trials. To evaluate the effects of aerosolised CPZ on capsaicin and citric acid induced cough, guinea pigs inhaled various

Abbreviations: ASIC, acid sensing ion channel; CPZ, capsazepine; I-RTX, iodo-resiniferatoxin; RAR, rapidly adapting receptor; TRPVI, transient receptor potential vanilloid-1 
concentrations of CPZ (30, 100, and $300 \mu \mathrm{M})$ before exposure to each tussigenic agent. Both the I-RTX and CPZ inhalation times were set at 10 minutes. In experiments aimed at evaluating the effects of I-RTX injected intraperitoneally (ip) on induced cough, guinea pigs were administered $0.03,0.1$, and $0.3 \mu \mathrm{mol} / \mathrm{kg}$ I-RTX 15 minutes before both capsaicin and citric acid inhalation. To determine the effects of ip CPZ on induced cough the animals were given $0.3,1$, and $30 \mu \mathrm{mol} / \mathrm{kg} \mathrm{CPZ} \mathrm{before} \mathrm{capsaicin} \mathrm{inhalation} \mathrm{or} 0.1,0.3$, and $1 \mu \mathrm{mol} / \mathrm{kg} \mathrm{CPZ}$ before inhalation of citric acid. In all experiments the effects of ip or aerosol administration of the I-RTX and CPZ vehicles (control conditions) on induced cough were also determined. Each animal received only one dose of antagonist.

To detect any non-specific inhibitory properties of ip I-RTX on the cough reflex, the ability of hypertonic saline $(7 \%$ sodium chloride, 1.2 M) to induce cough was also investigated. Hypertonic saline was administered for 10 minutes by aerosol 15 minutes after administration of the antagonist or its vehicle.

\section{Drugs}

Agents were obtained from the following companies: sodium chloride, citric acid, capsaicin, CPZ, (Sigma, Italy); I-RTX, (Tocris, UK). The stock concentrations of capsaicin (10 mM) and CPZ (10 mM) were prepared in 100\% ethanol. The stock concentration of I-RTX (1 mM) was prepared in $100 \%$ DMSO.

\section{Data analysis}

Values are presented as mean (SE). Comparisons between groups were made by one way analysis of variance (ANOVA) and the Student's $t$ test or the Bonferroni $t$ test when appropriate. A p value of $<0.05$ was considered significant. A minimum of eight guinea pigs was used to test the effect of the vehicle or of each single dose of the drugs. The inhibitory
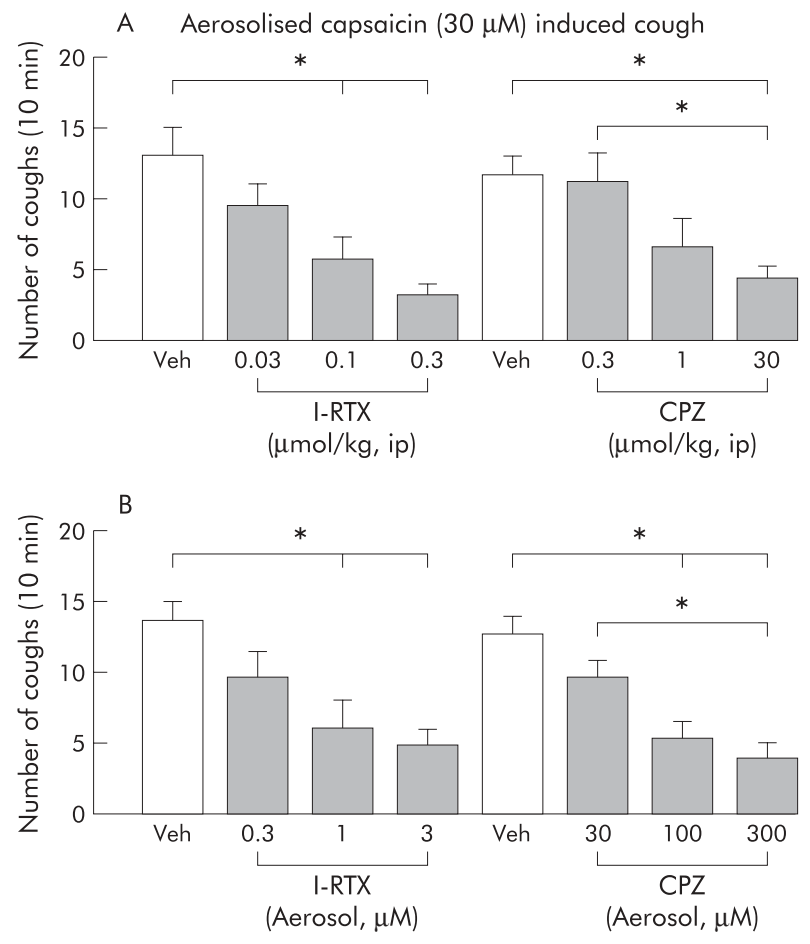

Figure 1 Inhibitory effect of (A) intraperitoneal (ip) and (B) aerosolised iodo-resiniferatoxin (I-RTX) or capsazepine (CPZ) on cough induced by capsaicin inhalation in guinea pigs. ${ }^{*} p<0.05 v$ vehicle (Veh). Each column represents the mean (SE) results of at least eight experiments.
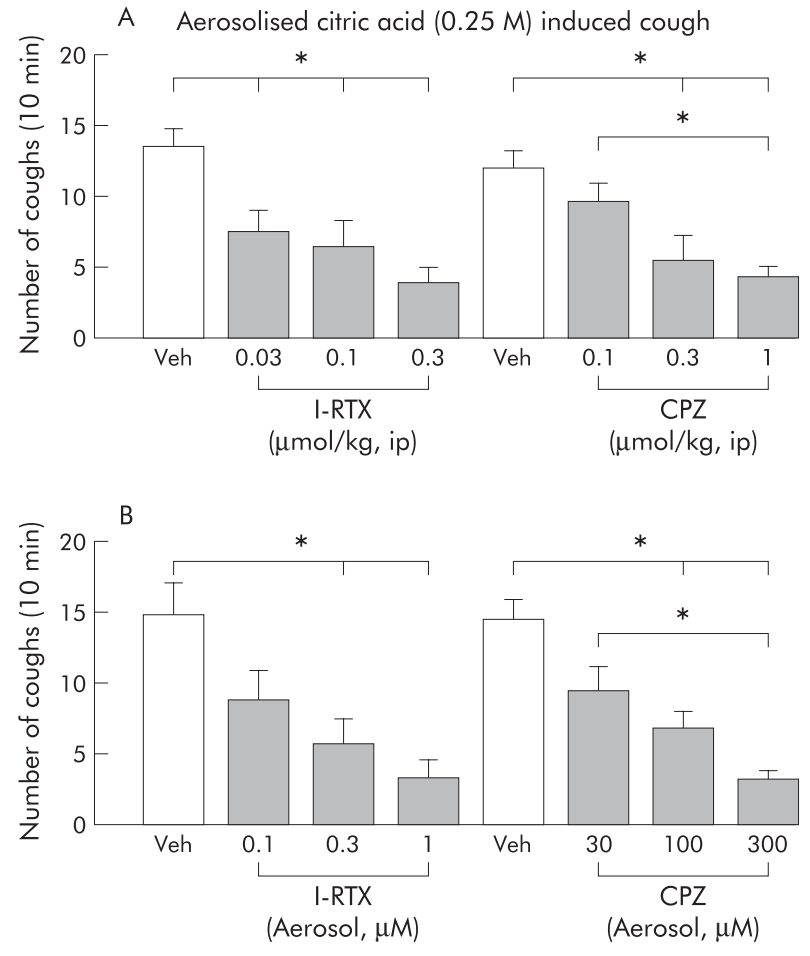

Figure 2 Inhibitory effect of (A) intraperitoneal (ip) and (B) aerosolised iodo-resiniferatoxin (I-RTX) or capsazepine (CPZ) on cough induced by citric acid inhalation in guinea pigs. ${ }^{*} p<0.05 v$ vehicle (Veh). Each column represents the mean (SE) results of at least eight experiments.

potency of I-RTX and CPZ was compared using the dose of antagonist that produces $50 \%$ inhibition $\left(\mathrm{ED}_{50}\right)$.

\section{RESULTS}

Independent of the route of administration, pretreatment with I-RTX and CPZ caused no obvious cough response in awake, freely moving guinea pigs. In no instance were inhalations of aerosolised CPZ or I-RTX followed by the appearance of even a few cough efforts. In contrast, inhalation of both capsaicin and citric acid (alone or in the presence of the CPZ or I-RTX vehicles) consistently caused a brisk tussive response in all animals tested.

The effects of pretreatment with I-RTX and CPZ on experimentally induced cough are shown in figs 1 and 2 . Compared with control conditions, ip and aerosol administration of I-RTX and CPZ consistently reduced the number of coughs provoked by inhalation of both capsaicin and citric acid $(\mathrm{p}<0.05$ in both sets of experiments). Furthermore, in all the experiments the inhibitory effects of I-RTX and CPZ on induced cough were dose dependent. Table 1 shows the $\mathrm{ED}_{50}$ values calculated after completion of the experiments. I-RTX administered either by aerosol or ip was significantly more potent than CPZ. Inhalation of hypertonic saline (7\% sodium chloride, 1.2 M) provoked a significant increase in the number of coughs (fig 3). Pretreatment with the highest dose of I-RTX $(0.3 \mu \mathrm{mol} / \mathrm{kg}$ ip $)$ used in this study did not affect the number of coughs induced by hypertonic saline inhalation.

\section{DISCUSSION}

The results show that intraperitoneal and aerosol administration of I-RTX is effective in reducing the number of coughs evoked by inhalation of both capsaicin and citric acid in guinea pigs. A previous investigation showed that I-RTX is a potent compound in antagonising capsaicin induced 
Table $1 \quad E D_{50}$ values of iodo-resiniferatoxin and capsazepine in inhibiting cough induced by capsaicin and citric acid in guinea pigs

\begin{tabular}{|c|c|c|c|c|}
\hline & \multicolumn{2}{|c|}{ lodo-resiniferatoxin } & \multicolumn{2}{|l|}{ Capsazepine } \\
\hline & $\begin{array}{l}\text { Aerosol } \\
\text { (nM) }\end{array}$ & $\begin{array}{l}\text { Intraperitoneal } \\
(\mathrm{nmol} / \mathrm{kg})\end{array}$ & $\begin{array}{l}\text { Aerosol } \\
(\mathrm{nM})\end{array}$ & $\begin{array}{l}\text { Intraperitoneal } \\
\text { (nmol/kg) }\end{array}$ \\
\hline $\begin{array}{l}\text { Capsaicin } \\
\text { Citric acid }\end{array}$ & $\begin{array}{l}284(92)^{*} \\
84(19)^{*}\end{array}$ & $\begin{array}{l}29(9)^{*} \\
16(4)^{*}\end{array}$ & $\begin{array}{l}34280(867) \\
12840(1621)\end{array}$ & $\begin{array}{l}860(27) \\
150(6)\end{array}$ \\
\hline
\end{tabular}

contractions in isolated bronchial rings and strips of guinea pig urinary bladder. ${ }^{20}$ The contractile response produced by capsaicin in these tissues results from smooth muscle contraction secondary to tachykinin release from terminals of primary sensory neurons following stimulation of TRPV1. We suggest that the inhibitory effect of I-RTX on capsaicin induced cough in guinea pigs is also caused by its ability to antagonise the activation of TRPVI. It should be emphasised, however, that the pharmacological characterisation of I-RTX as a TRPVl antagonist is far from complete. Previous evidence indicated that I-RTX is a powerful antagonist at TRPVI in vitro and in vivo. ${ }^{19}{ }^{20}$ However, later studies confirmed the high potency of I-RTX in vitro but not in vivo in capsaicin induced paw flinching in rats. ${ }^{21}$ In this latter study I-RTX produced some excitatory effects at the highest doses. Likewise, minor excitation was produced by I-RTX when injected intraperitoneally to induce the writhing response. ${ }^{20}$ It is possible that a minor agonistic component is retained by IRTX and is unmasked when the drug is administered locally in a circumscribed environment (intraperitoneally, subcutaneously), but not following systemic administration. ${ }^{19} 20$ Thus, the possibility that I-RTX retains some agonistic activity and that an agonist-dependent desensitising effect on sensory nerves contributes to its sensory neuron blocking activity cannot be completely discounted. In contrast, in none of our experiments did I-RTX cause a tussive response. One possible explanation for this is that inhalation of the drug causes widespread diffusion of the molecules into the airways so that its minor (if any) agonistic activity is minimised while its antagonist potential is maintained.

Citric acid is thought to induce cough by its ability to donate protons that are powerful stimulants of different subpopulations of primary sensory neurons including the rapidly adapting receptors (RAR) - for example, the subtype that plays a major role in the tussive response. ${ }^{22}$ Protons may stimulate neurons with $\mathrm{C}$ and $\mathrm{A}-\delta$ fibres by activating different channels, two of which have been well characterised: (1)

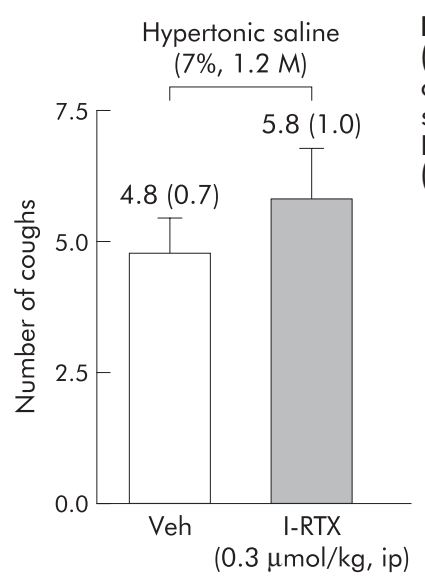

the TRPVI ${ }^{3}{ }^{4}$ and (2) the acid sensing ion channels (ASICs). ${ }^{23}$ It has been suggested that RARs do not express TRPVI, ${ }^{22}$ which suggests that ASIC ought to be responsible for the cough induced by citric acid. However, a previous study showed that CPZ was able to inhibit citric acid induced cough. ${ }^{11}$ Since CPZ is not a high affinity antagonist of TRPVl, this finding may raise doubts as to its selectivity in inhibiting the cough response. Nevertheless, the findings of Lalloo et al ${ }^{11}$ suggested that TRPVI rather than ASIC is responsible for citric acid induced cough. The present findings strongly support this view as I-RTX, a TRPVI antagonist chemically unrelated to $\mathrm{CPZ}$, is also able to inhibit citric acid induced cough effectively. However, a small component of the cough response to citric acid remained even at the highest doses of both I-RTX and CPZ. This residual response might be due to stimulation by citric acid of a non-TRPVl channel such as the ASIC. Alternatively, it may still be caused by TRPVI activation due to partial blockade of the channel by the two antagonists.

Capsaicin induced cough was also not completely inhibited by I-RTX or CPZ. Since it is presumed that the cough response induced by capsaicin is entirely mediated by TRPVI, it is likely that the incomplete abolition of citric acid induced cough by CPZ and I-RTX is caused by the partial blockade of TRPVI at the doses of the antagonists used in the present study. An alternative explanation is that splice variants such as those described in the rat ${ }^{24}{ }^{25}$ also occur in guinea pigs, and these variants could exhibit different affinities for different agonists. In addition, although the expression in recombinant systems suggests that the channel monomer is sufficient for the pore function of TRPVl, there is evidence that TRPVI is capable of forming a specific ternary complex with phospholipase $\mathrm{C}$ and the neurotrophin trkA receptor. ${ }^{26}$ These differences in assembling the channel may be tissue specific and may result in different affinities of antagonists.

Finally, recent electrophysiological observations ${ }^{27}$ in single airway neurons of guinea pigs show that nerve activation following exposure to acid occurs in two different ways: a slowly inactivating mechanism present in $\mathrm{C}$ fibres which is TRPVI dependent as it is blocked by CPZ and I-RTX, and a rapidly inactivating mechanism present in A- $\delta$ fibres which acts independently of TRPVI. The pharmacological observation that vanilloid receptor antagonism (present findings) inhibits most of the tussive response to citric acid ${ }^{11}$ leads to the surprising conclusion that the TRPVI resistant pathway has a minor role in acid induced cough in guinea pigs.

Another important observation of the present study is that I-RTX was several times more potent than CPZ in reducing the cough response to capsaicin and citric acid. In vitro assays in different mammalian species have shown that I-RTX has a very high potency towards TRPVl, being 100-1000 times more potent than CPZ. ${ }^{19}{ }^{20}$ This striking difference in vitro between I-RTX and CPZ has been confirmed in vivo in the mouse and rat where neurogenic plasma extravasation and nociceptive responses such as the writhing test ${ }^{20}$ or the capsaicin pain test have been studied. ${ }^{19}$ The finding that 
I-RTX was 10-100 times more potent than CPZ in inhibiting experimentally induced cough is therefore in keeping with the results of previous in vivo investigations. ${ }^{1920}$ The observation that both CPZ and I-RTX were more effective (2-5-fold) in inhibiting cough induced by citric acid than cough induced by capsaicin further supports the view that citric acid cough is sensitive to TRPVl antagonism.

The overall importance of TRPVl in human cough is not fully understood. Available evidence suggests that TRPVI is of considerable importance because stimuli that activate these channels are powerful tussive agents. In disease states the sensitivity of TRPVI may be upregulated by proinflammatory mediators including bradykinin and nerve growth factor $^{28} 29$ which have an important role in cough and asthma. $^{30}$ The threshold dose of capsaicin to induce cough in patients with asthma and $\mathrm{COPD}^{13}{ }^{14}$ is lowered, and patients with gastro-oesophageal reflux often suffer from cough. A high affinity and selective TRPVl antagonist is needed to show the pathophysiological role of TRPVI in the cough experienced by these different groups of patients.

In conclusion, we have shown that I-RTX, a novel and ultra potent antagonist at the native rat and guinea pig TRPVI and at the recombinant human TRPVl, inhibits cough mediated by agents possibly acting via TRPVI stimulation. I-RTX can therefore be considered as an exemplar for the design of novel antitussive agents.

\section{Authors' affiliations}

M Trevisani, A Milan, R Gatti, S Harrison, P Geppetti, Center of Excellence for the Study of Inflammation, University of Ferrara, Ferrara, Italy

A Zanasi, Department of Thoracic-Pumonary Diseases, Unit of Respiratory Physiopathology, University of Bologna, Bologna, Italy G Fontana, P Geppetti, Department of Critical Care Medicine and Surgery, University of Florence, Florence, Italy

A H Morice, Academic Medicine, University of Hull, Hull, UK

This work was supported in part by ARCA, Padua and MUIR, Rome.

\section{REFERENCES}

1 Caterina MJ, Schumacher MA, Tominaga M et al. The capsaicin receptor: a heat-activated ion channel in the pain pathway. Nature 1997;389:816-24.

2 Benham CD, Davis JB, Randall AD. Vanilloid and TRP channels: a family of lipid-gated cation channels. Neuropharmacology 2002;42:873-88.

3 Tominaga $M$, Caterina MJ, Malmberg $A B$, et al. The cloned capsaicin receptor integrates multiple pain-producing stimuli. Neuron 1998;21:531-43.

4 Bevan S, Geppetti P. Protons: small stimulants of capsaicin-sensitive sensory nerves. Trends Neurosci 1994; 17:509-12

5 Zygmunt PM, Petersson J, Andersson DA, et al. Vanilloid receptors on sensory nerves mediate the vasodilator action of anandamide. Nature 1999;400:452-7.
6 Hwang SW, Cho H, Kwak J, et al. Direct activation of capsaicin receptors by products of lipoxygenases: endogenous capsaicin-like substances. Proc Natl Acad Sci USA 2000;97:6155-60.

7 Huang SM, Bisogno T, Trevisani M, et al. An endogenous capsaicin- like substance with high potency at recombinant and native vanilloid VR I receptors. Proc Natl Acad Sci USA 2002;99:8400-5.

8 Forsberg K, Karlsson JA. Cough induced by stimulation of capsaicin-sensitive sensory neurons in conscious guinea-pigs. Acta Physiol Scand $1986 ; 128: 319-20$

9 Midgren B, Hansson L, Karlsson JA, et al. Capsaicin induced cough in humans. Am Rev Respir Dis 1992;146:347-51.

10 Morice AH, Kastelik JA, Thompson R. Cough challenge in the assessment of cough reflex. Br J Clin Pharmacol 2001;52:365-75.

11 Lalloo UG, Fox AJ, Belvisi MG, et al. Capsazepine inhibits cough induced by capsaicin and citric acid but not by hypertonic saline in guinea pigs. J Appl Physiol 1995;79: 1082-7.

12 Kastelik JA, Thompson RH, Aziz I, et al. Sexrelated differences in cough reflex sensitivity in patients with chronic cough. Am J Respir Crit Care Med 2002;166:961-4.

13 Doherty MJ, Mister R, Pearson MG, et al. Capsaicin responsiveness and cough in asthma and chronic obstructive pulmonary disease. Thorax 2000;55:643-9.

14 Wong $\mathrm{CH}$, Morice $\mathrm{AH}$. Cough threshold in patients with chronic obstructive pulmonary disease. Thorax 1999:54:62-4.

15 O'Connell F, Thomas VE, Pride NB, et al. Capsaicin cough sensitivity decreases with successful treatment of chronic cough. Am J Respir Crit Care Med 1994; 150:374-80.

16 Maggi CA, Bevan S, Walpole CS, et al. A comparison of capsazepine and ruthenium red as capsaicin antagonists in the rat isolated urinary bladder and vas deferens. Br J Pharmacol 1993;108:801-5.

17 Szallasi A. The vanilloid (capsaicin) receptor: receptor types and species differences. Gen Pharmacol 1994;25:223-43.

18 Szallasi A, Blumberg PM. Vanilloid (capsaicin) receptors and mechanisms. Pharmacol Rev 1999;51:159-212.

19 Wahl P, Foged C, Tullin S, et al. lodo-resiniferatoxin, a new potent vanilloid receptor antagonist. Mol Pharmacol 2001;59:9-15.

20 Rigoni M, Trevisani M, Gazzieri D, et al. Neurogenic responses mediated by vanilloid receptor-1 (TRPV1) are blocked by the high affinity antagonist, iodoresiniferatoxin. Br J Pharmacol 2003;138:977-85.

21 Seabrook GR, Sutton KG, Jarolimek W, et al. Functional properties of the high-affinity TRPVI (VR1) vanilloid receptor antagonist (4-hydroxy-5-iodo-3methoxyphenylacetate ester) iodo-resiniferatoxin. J Pharmacol Exp Ther 2002;303:1052-60.

22 Widdicombe JG. Afferent receptors in the airways and cough. Respir Physiol 1998; 114:5-15.

23 Chen CC, England S, Akopian AN, et al. A sensory neuron-specific, proton gated ion channel. Proc Natl Acad Sci USA 1998;95:10240-5.

24 Xue $Q, Y u$ Y, Trilk SL, et al. The genomic organization of the gene encoding the vanilloid receptor: evidence for multiple splice variants. Genomics 2001;76:14-20.

25 Sanchez JF, Krause JE, Cortright DN. The distribution and regulation of vanilloid receptor VR1 and VR1 5' splice variant RNA expression in rat. Neuroscience 2001;107:373-81.

26 Gunthorpe MJ, Benham CD, Randall A, et al. The diversity in the vanilloid (TRPV) receptor family of ion channels. Trends Pharmacol Sci 2002;23:183-91.

27 Kollarik M, Undem BJ. Mechanisms of acid-induced activation of airway afferent nerve fibres in guinea-pig. J Physiol 2002;543:591-600.

28 Premkumar LS, Ahern GP. Induction of vanilloid receptor channel activity by protein kinase C. Nature 2000;408:985-90

29 Chuang $\mathrm{HH}$, Prescott ED, Kong $\mathrm{H}$, et al. Bradykinin and nerve growth factor release the capsaicin receptor from Ptdlns(4,5)P2-mediated inhibition. Nature 2001;411:957-62.

30 Bonini S, Lambiase A, Properzi F, et al. Nerve growth factor and asthma. Allergy 2002;72:13-5. 